\title{
The membrane shape mapping of the artificial ventricle in the actual dimensions
}

\author{
Wojciech Sulej \\ Military University of Technology \\ Kaliskiego Str. 2, \\ 01-489 Warsaw, Poland, \\ Email: wojciech.sulej@wat.edu.pl
}

\author{
Krzysztof Murawski \\ Military University of Technology \\ Kaliskiego Str. 2, \\ 01-489 Warsaw, Poland, \\ IEEE Member \# 92707852 \\ Email: krzysztof.murawski@wat.edu.pl
}

\begin{abstract}
The paper sets out and presents a new approach to determine the shape of the flaccid membrane of the extracorporeal pneumatic heart assist pump. This is a continuation of earlier work on the use of image processing and analysis techniques to determine the membrane shape of an artificial ventricle. The study focused on the membrane shape mapping in the actual dimensions - in the real world. The method to transform measurement results in pixels to dimensions in the real world in millimetres as well as the obtained results of this process were presented.
\end{abstract}

\section{INTRODUCTION}

$\mathrm{T}$ HIS paper presents how to use a new technique, the Depth From Defocus (DFD) type presented in [1 - 5], to determine the shape of the flaccid membrane of the extracorporeal pneumatic heart assist pump, Fig. 1, in the actual dimensions - in the real world. The work carried out in this area provides an opportunity to develop a method for determining the stroke volume of the artificial ventricle. Studies of this type are carried out in the framework of the Polish Artificial Heart (PSS) [6 - 8]. As a result of the lack of satisfactory solutions original works are suspended. Thus, to solve the problem, studies on a visual method of the measurement were initiated; e.g. [9-12]. Additionally this method creates the possibility of a practical use of opaque biologically inert layers, which are already developed in the framework of PSS. This should significantly reduce the risk of the formation of clots while maintaining the safe operation of the heart support pump.

\section{MOTIVATION}

One of the basic heart function parameters is the stroke volume of the chamber. The instantaneous stroke volume of

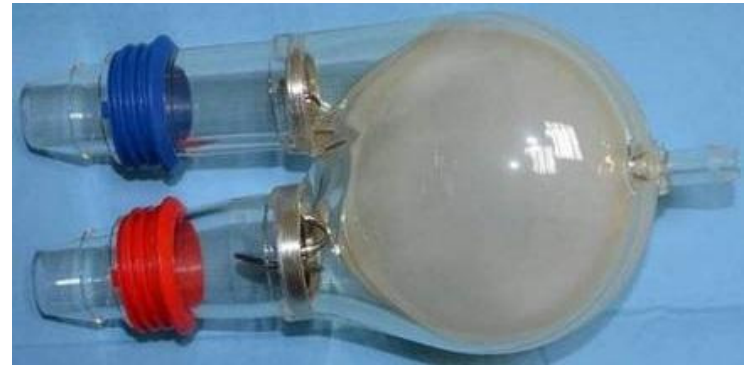

Fig. 1 The extracorporeal pneumatic heart assist pump developed in the framework of the Polish Artificial Heart a controlled pneumatic artificial ventricular can be determined by knowing the momentary shape of its flaccid membrane. Presented in [1 - 5] the Depth From Defocus (DFD) type method has been developed for visual distance measuring. It can also successfully be used for the construction of the sensor defining the shape of the flaccid membrane of the extracorporeal heart support pulse pump. It can take two forms of implementation. Until now the visualization of the shape of the membrane was performed only in the virtual world (augmented reality). Currently, studies are being carried out to lead to the determination of the shape of the membrane in the real world. Mapping the membrane shape of the artificial ventricle in the actual dimensions will enable the user to determine the stroke volume using the numerical integration method.

\section{OBJECT OF THE STUDY}

The study was conducted on the extracorporeal pneumatic heart assist pump model, Fig. 2. The model was modelled based on the ReligaHeart ${ }^{\circledR}$ EXT prosthetic, Fig. 1. Using the model is justified because of the significant costs of the original prosthesis. The pneumatic heart assist pump model has a pneumatic chamber and a blood chamber separated by a flaccid membrane. The momentary shape of the flaccid membrane affects the volume of the blood chamber. The membrane is controlled by air into the pneumatic chamber. Pushing out air into the pneumatic chamber results in movement of the membrane downward. This state corresponds to the ejection of fluid from the blood chamber.

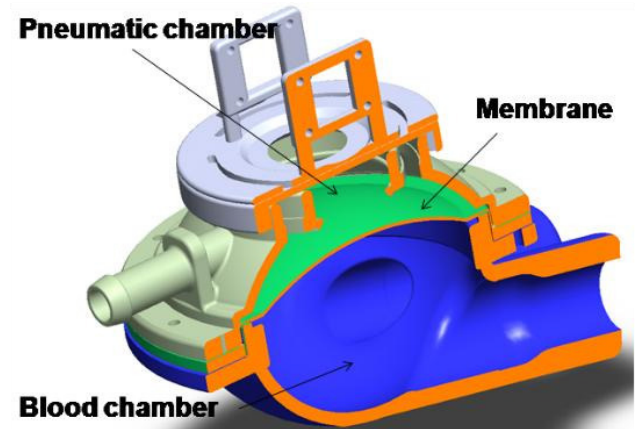

Fig. 2 The cross-section of the extracorporeal pneumatic heart assist pump mode 
The opposite process causes the rising of the membrane and filling bloody chamber with liquid.

Using a flaccid membrane in the pneumatic heart assist pump, even though raises many problems, is necessary from a medical point of view. Such a membrane limits the formation of coagulation and eliminates the problem of sedimentation of blood (dividing into fractions).

\section{MEASUREMENT METHOD}

The essence of the used measurement method is to observe a surface of the membrane at a close distance with a fixed-focus camera equipped with wide-angle lens and to determine the shape of this membrane in the 3-dimensional space on the basis of a one-shot image.

In the measurement process the image processing and analysis techniques are used. Firstly, the image is acquired from the camera. Next, the image is masked using a circle mask in order to hide unnecessary parts of the image. Then the image segmentation using thresholding is performed. After that, markers are detected. This method works due to markers (in the study, round white markers with a diameter of $3 \mathrm{~mm}$ ) arranged on the surface of the membrane from the pneumatic chamber side. During the membrane movement the markers do not change their physical size. Their smaller or larger surface area visible in the image is related only to their proximity or distance from the front of the camera. On the basis of pixel coordinates and a surface area of each marker we can determine their location in the 3-dimensional space. Having the determined several dozen markers, the same number of real points on the membrane surface can be obtained. Other points, in the required quantity, are determined using triangulation-based cubic interpolation.

As a result, a grid representing the membrane shape of the artificial ventricle in the actual dimensions is obtained. This method is very fast by the fact that during measurement the position of the camera and all lens and camera settings (focus, aperture and focal length) remain unchanged.

\section{THE DIMENSIONS OF THE MEMBRANE}

The difficulty of determining the real membrane shape of the heart assist pump fitted with a flaccid membrane, Fig. 3, is that the pump has only two membrane states with a known mathematical description. The first state occurs when the blood chamber is fully submerged with blood; the membrane then takes on a convex shape. The second state occurs with full blood pressure from the heart pump; the membrane then takes on a concave shape.

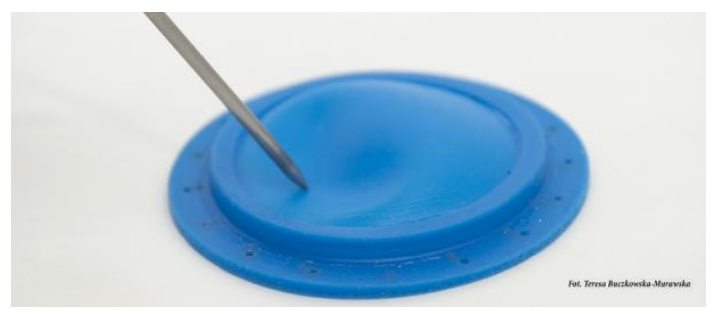

Fig. 3 The flaccid membrane

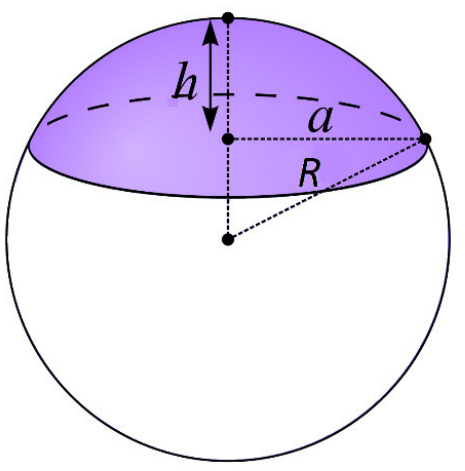

Fig. 4 The geometric dimensions of the spherical cap

In both these states the membrane takes on a shape of the spherical cap, Fig. 4. For these characteristic states the geometric dimensions can be determined because a few parameters of the pump designed in the framework of PSS are given. The radius of the spherical cap $a$ equals $35 \mathrm{~mm}$. The volume of this spherical cap $V$ equals $35 \mathrm{ml}$ and this is a half of $70 \mathrm{ml}$ which is the assumed stroke volume of a heart for an adult man. With the known radius of the spherical cap the geometrical dimensions of the membrane on the $\mathrm{X}-\mathrm{Y}$ plane are known. To determine the extreme positions of the membrane in the Z-axis the value of $h$ is required. It can be determined on the basis of the formula (1) and after solving the equation (2).

$$
\begin{aligned}
& V=\left(\left(\pi h^{2}\right) / 3\right) \cdot(3 R-h) \\
& h^{3}+3 a^{2} h-6 V / \pi=0
\end{aligned}
$$

The calculated value of $h$ equals $16.8803 \mathrm{~mm}$. Having values of $a$ and $h$ the all extreme positions of the membrane in the 3-dimensional space are known.

\section{TRANSFORMATION OF THE X- AND Y-COORDINATES}

The transformation of the $\mathrm{x}$ - and $\mathrm{y}$-coordinates is performed directly based on the pixel coordinates of the image, Fig. 5.

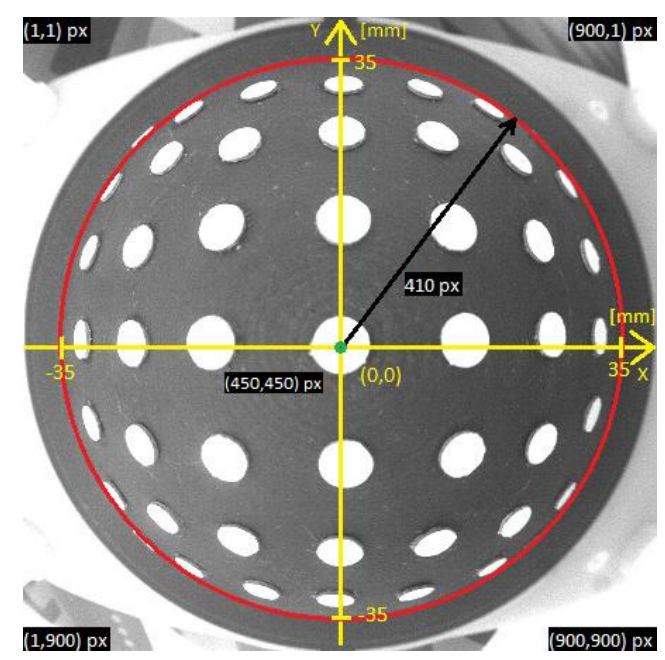

Fig. 5 View of the membrane from camera with the coordinate system added 
For transformation purpose, the 2-dimensional shape of the membrane determined in pixels is calculated using a linear transformation for the x-coordinate (3) and for the $y$ coordinate (4).

$$
\begin{aligned}
& X=\left[\left(x-x_{0}\right) /(2 \cdot r)\right] \cdot\left(X_{\max }-X_{\text {min }}\right) \\
& Y=\left[\left(y_{0}-y\right) /(2 \cdot r)\right] \cdot\left(Y_{\text {max }}-Y_{\text {min }}\right)
\end{aligned}
$$

In the study, a 900 px x 900 px image, masked by a circle of a radius $r=410 \mathrm{px}$, is calculated to a range from $-35 \mathrm{~mm}$ to $35 \mathrm{~mm}$, assuming that the centre of the scale is in the centre of the membrane. For the givens:

$$
\begin{aligned}
& r=410 p x, \quad x_{0}=450 p x, \quad y_{0}=450 p x, \\
& X_{\text {max }}=Y_{\text {max }}=35 \mathrm{~mm}, \quad X_{\text {min }}=Y_{\text {min }}=-35 \mathrm{~mm}
\end{aligned}
$$

We obtain the simplified linear transformation equations:

$$
\begin{aligned}
& X=0.08537 \cdot x-38.4146[\mathrm{~mm}] \\
& Y=-0.08537 \cdot \mathrm{y}+38.4146[\mathrm{~mm}]
\end{aligned}
$$

The equations (5) and (6) can be used to the transformation of the $\mathrm{x}$ - and $\mathrm{y}$-coordinates from pixels to millimetres. So far a grid defined in such a way presents the determined shape of the membrane dimensioned in the actual 2-dimensional space.

\section{TRANSFORMATION OF THE Z-COORDINATE}

The transformation of the $\mathrm{z}$-coordinate is not performed directly based on the pixel coordinates of the image. To solve the problem, the dependency of the marker area from a distance of the marker to the front of the camera must be determined. This dependency is not constant even for the same artificial ventricle model and will vary due to various factors. The camera and lens parameters, the type of lighting, the threshold value, size and colour of markers, kind of surface and colour of the membrane can affect this relationship. Trial tests confirmed that depending on the mentioned factors, different values of the marker area in the image can be obtained for the same distance. This applies in particular to the central marker which, due to its location, can achieve the largest and the smallest possible surface area in the image and thus determines the extreme values in the Z-axis. Simultaneously it can observe that during changing

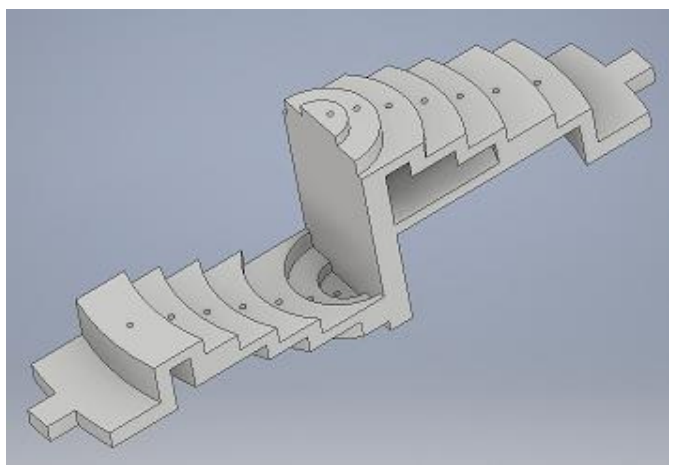

Fig. 6 The measurement pattern designed in CAD software

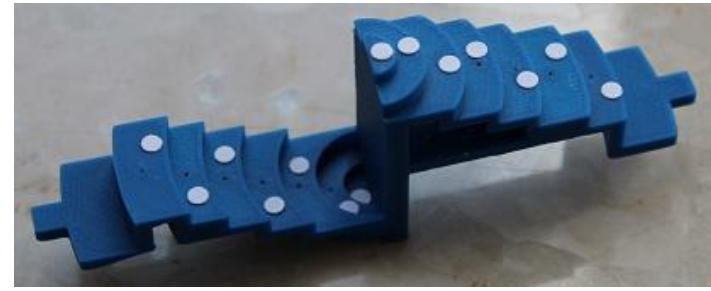

Fig. 7 The ready-made measurement pattern

working conditions of the heart pump, the proportion of the changes of a surface area of the markers at different distances remains constant. This leads to the conclusion that the differences between the markers area at different distances can have a linear character. In order to verify this hypothesis the suitable measurement pattern was designed, Fig. 6. This takes the form of steps of well-known heights. The $3 \mathrm{~mm}$ difference between steps (except the extremes) was assumed. The pattern also allows to compare the measurement results with the reference distances of each marker to the front of the camera optical sensor; e.g. for the calibration purpose. The measurement pattern was designed in CAD software. Then it was 3D printed with an accuracy of $0.001 \mathrm{~mm}$ on the $\mathrm{X}$ and $\mathrm{Y}$ axes with a layer thickness on the Z-axis of $0.09 \mathrm{~mm}$, Fig. 7. On the steps of the pattern 14 round, white, markers having a diameter of $3 \mathrm{~mm}$ were arranged, one on each step.

For the study, a laboratory stand was designed and built. Different parts of the stand were printed on a $3 \mathrm{D}$ printer. After assembly a stable structure was created eliminating random movements and changes in camera viewing angles with respect to the membrane or the pattern in each of the spatial dimensions, Fig. 8. The station allows for quick and easy replacement of the tested membranes or patterns. It is equipped with a miniature monochrome XIMEA camera model MU9PM-MH with a lens with a fixed focal length $f=1.8 \mathrm{~mm}$ and a viewing angle of $126^{\circ}$. The camera is connected to a computer using a USB 2.0 port interface. This is completed with the authors' software, which enables measurement and enables real-time imaging.

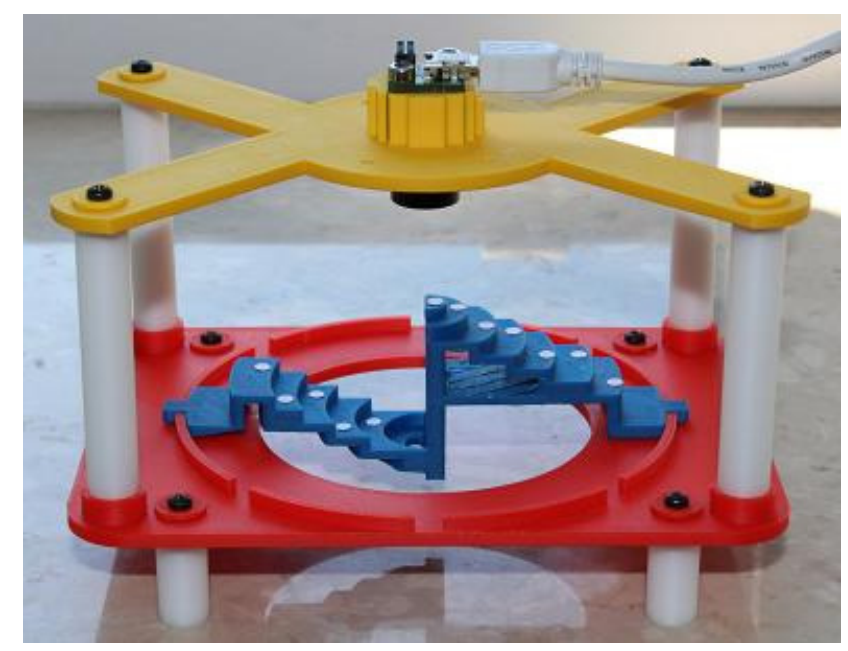

Fig. 8 The laboratory stand with the measurement pattern 


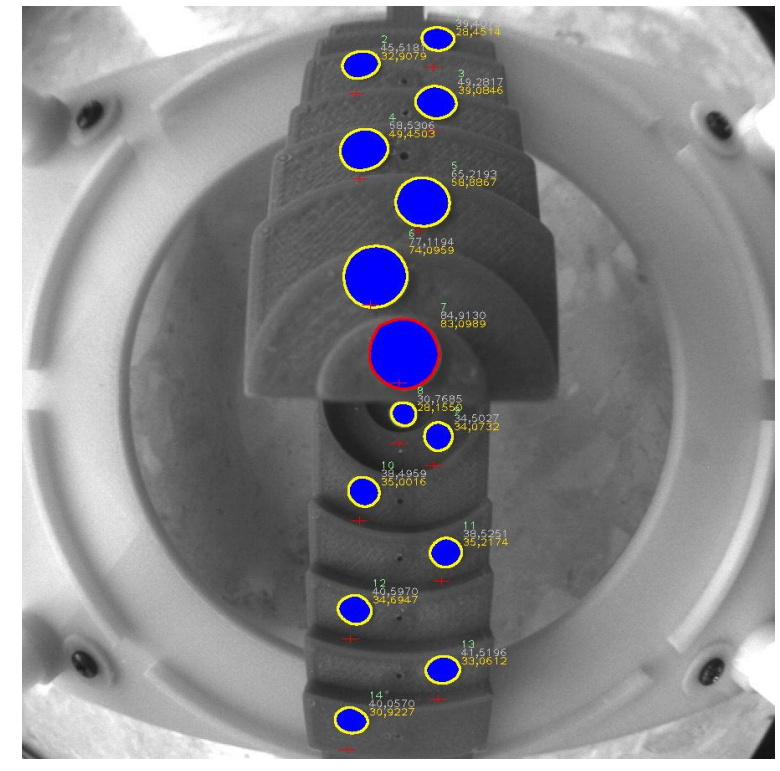

Fig. 9 View of the measurement pattern from camera with detected markers

The pattern was mounted into the laboratory station and the measurement procedure was performed, Fig. 9. The measurement was conducted in three different conditions changing lighting parameters and the threshold value. The obtained values of a surface area of the detected markers for all three cases have been collected in Table I. At the same time, a surface area of all markers at different heights was determined. The height (distance) equal to 0 was assumed on the level of the outline of a circular membrane. The image sharpness was set on this level focusing on the central marker. For a distance greater than 0 (a height less than 0), the appropriate negative value was assumed.

TABLE I.

THE DEPENDENCY OF THE MARKER AREA FROM THE HEIGHT

\begin{tabular}{|r|r|r|r|}
\hline \multirow{2}{*}{$\begin{array}{c}\text { Height } \\
{[\mathbf{m m}]}\end{array}$} & \multicolumn{3}{|c|}{ Marker area [px] } \\
\cline { 2 - 4 } & E. 1 & E. 2 & E. 3 \\
\hline 16.8803 & 6156 & 5663 & 4844 \\
\hline 15 & 5762 & 5271 & 4479 \\
\hline 12 & 4938 & 4141 & 3447 \\
\hline 9 & 3788 & 3391 & 2798 \\
\hline 6 & 3045 & 2607 & 2103 \\
\hline 3 & 2189 & 1777 & 1347 \\
\hline 0 & 1439 & 1039 & 545 \\
\hline-3 & 1357 & 1054 & 486 \\
\hline-6 & 1292 & 894 & 436 \\
\hline-9 & 1219 & 866 & 404 \\
\hline-12 & 1169 & 864 & 391 \\
\hline-15 & 1067 & 685 & 374 \\
\hline-16.8803 & 954 & 744 & 349 \\
\hline
\end{tabular}

where E. $n$ is a number $(n)$ of experiment

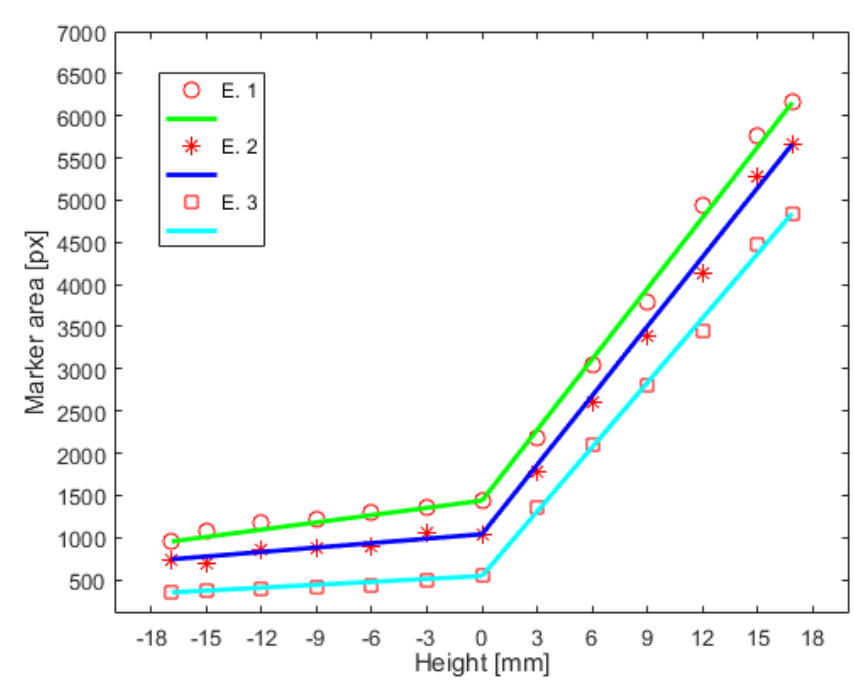

Fig. 10 Graphs of the dependency of the marker area from the height

The results confirmed that the differences between the markers area at different distances have a linear character. However, the graph of the dependency of the marker area from the height, Fig. 10, shows that two separate dependencies are needed for each case. The approximation of results shown that for the $\mathrm{z}$-coordinate transformation purpose, it is not necessary to determine a dependency of the marker area from the distance for all possible values, but is enough to measure only two extremes and to know a value of zero from the calibration. This should significantly simplify the sensor calibration and to allow making necessary corrections even during normal operation of the pneumatic heart assist pump.

Assuming that $A_{z}$ is a surface area of the current marker, $A_{\text {zero }}$ is a surface area of the central marker on the level of the outline of a circular membrane, $A_{t o p}$ is a surface area of the central marker for the membrane in the maximum upper position and $A_{\text {bottom }}$ is a surface area of the central marker for the membrane in the maximum down position, the equations $(7-9)$ for the $\mathrm{z}$-coordinate were determined.

$$
\begin{aligned}
& z=A_{z}-A_{\text {zero }} \\
& Z=\left(z \cdot Z_{\text {max })}\right) /\left(A_{\text {top }}-A_{\text {zero }}\right) \quad \text { for } z \geq 0 \\
& Z=\left(z \cdot Z_{\text {min }}\right) /\left(A_{\text {bottom }}-A_{\text {zero }}\right) \text { for } z<0
\end{aligned}
$$

In the study, for the givens:

$$
\begin{gathered}
A_{\text {top }}=6156 p x, A_{\text {zero }}=1439 p x, A_{\text {bottom }}=954 p x, \\
Z_{\text {max }}=16.8803 \mathrm{~mm}, \quad Z_{\text {min }}=-16.8803 \mathrm{~mm}
\end{gathered}
$$

We obtain the simplified linear transformation equations:

$$
\begin{aligned}
& Z=0.00358 \cdot A_{z}-5.1496[\mathrm{~mm}] \text { for } A_{z} \geq 1439 \\
& Z=0.0348 \cdot A_{z}-50.0843[\mathrm{~mm}] \text { for } A_{z}<1439
\end{aligned}
$$

The equations (10) and (11) can be used to the transformation of the z-coordinate. Finally, using also the previously determined equations (5) and (6), a grid defined in such a way presents the determined shape of the membrane dimensioned in the actual 3-dimensional space. 


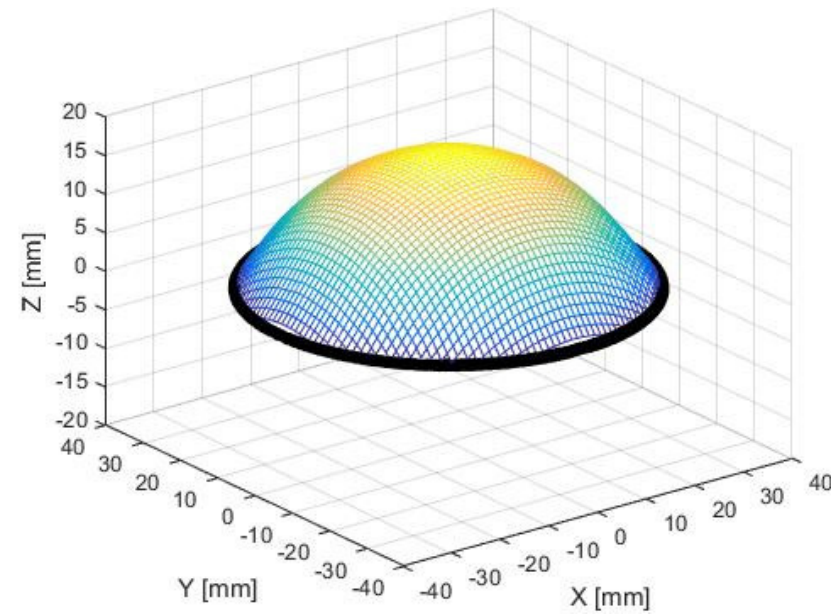

Fig. 11 The ideal shape of convex membrane in the actual dimensions

\section{RESULTS OF RESEARCH}

Knowing the suitable transformation equations, the shape of the flaccid membrane of the extracorporeal pneumatic heart assist pump model in the actual dimensions was possible to determine. Firstly, the ideal shapes of convex and concave membrane, Fig. 11 and Fig. 12, in the actual dimensions were determined based on the geometric dimensions of the spherical cap. Then the full measurement procedure was performed for these extreme positions of the membrane, Fig. 13 and Fig. 14. As a result, it was possible to obtain the shape mapping of the membrane in the form of a measuring grid and determining for each point of the grid a reference value. With these two values the measurement errors could be determined and their causes analyzed. Obtained from the measurements, mappings of a shape of convex and concave membrane, Fig. 15 and Fig. 16, in the actual dimensions have confirmed a propriety of the determined transformation equations.

On the surface of the membrane 49 round, white, markers having a diameter of $3 \mathrm{~mm}$ were arranged. The study assumed an even distribution of markers forming squares. The distance between the centres of the neighbouring markers was $7.7 \mathrm{~mm}$. Markers were placed starting from the

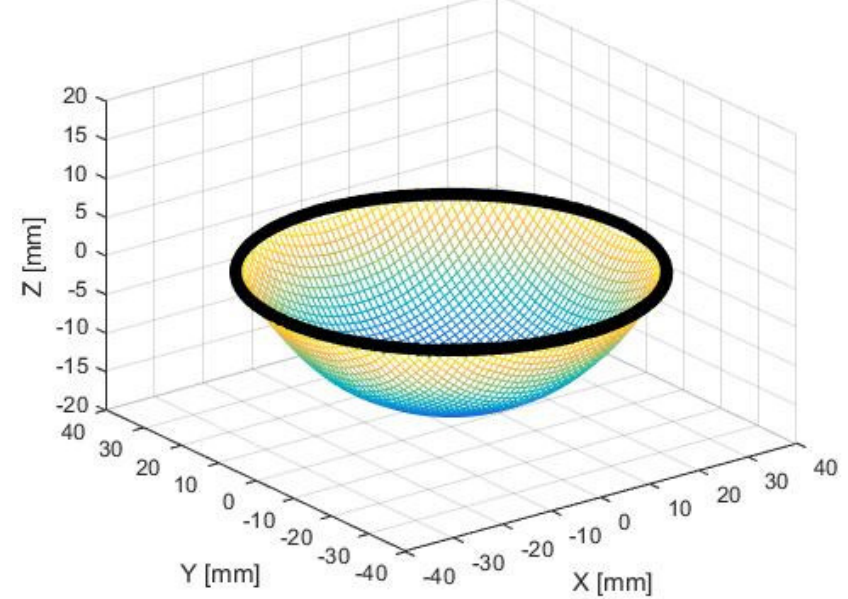

Fig. 12 The ideal shape of concave membrane in the actual dimensions

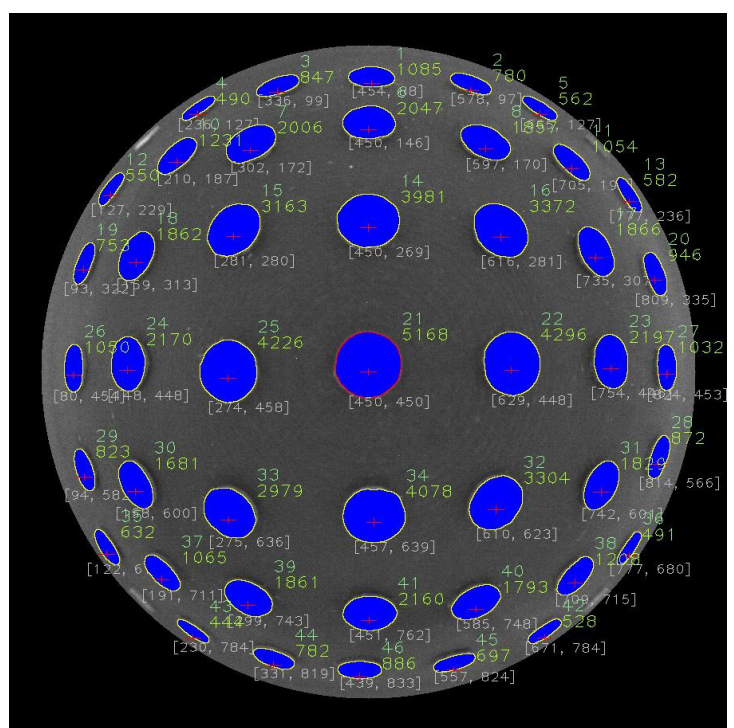

Fig. 13 View from camera of convex membrane with detected markers

central marker. Analyzing the results, deformation of the membrane shape around the markers' positions is noticeable. This is due to the interpolation that is used to calculate values between markers.

In the case with a convex membrane the mapping of the membrane shape is appropriate. The achieved shape, almost on the entire surface, is comparable to that of the ideal membrane. For the concave membrane the mapping of the membrane assumes the correct shape of a spherical cap. The greatest errors were obtained for the markers, which are far from the centre of the membrane.

In both cases difference between all of the measurement values and their reference values does not exceed 5\%. The shape of the projection is subject to the smallest error in the vicinity of the central marker. The biggest inaccuracy was obtained for markers which position was close to the edge of the membrane. This effect is mainly due to insufficient number of markers located at these places.

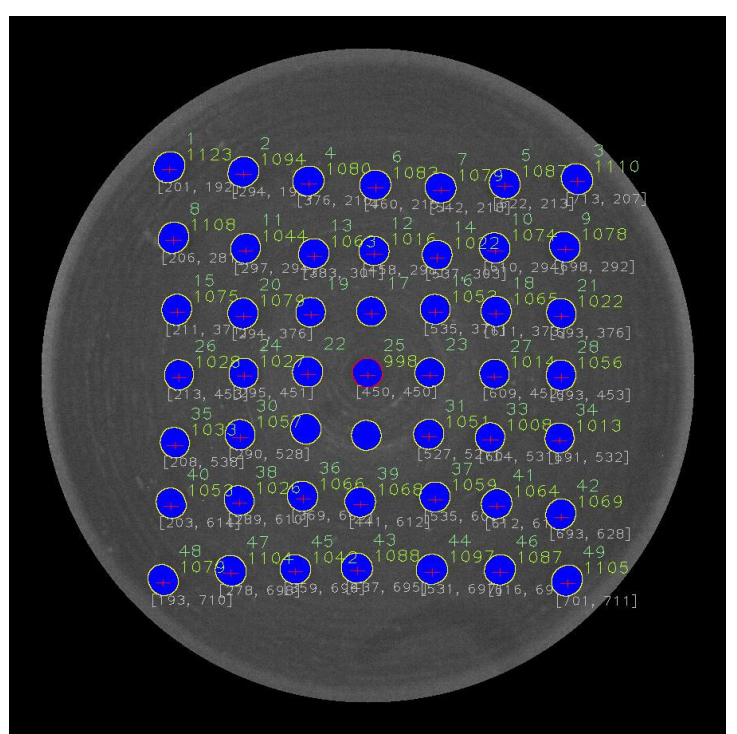

Fig. 14 View from camera of concave membrane with detected markers 


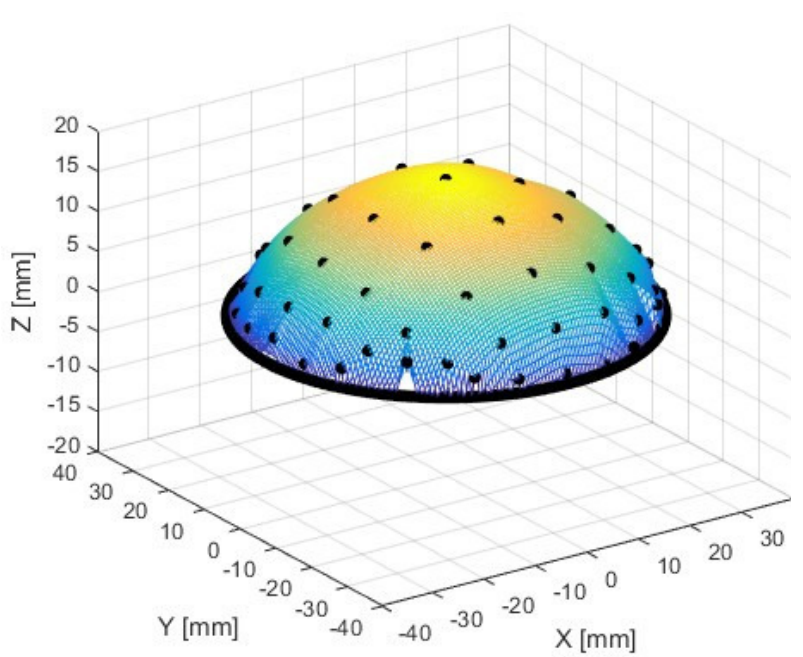

Fig. 15 The mapped shape of convex membrane from the measurements

\section{CONCLUSION}

The paper presents a method of the membrane shape mapping of the extracorporeal pneumatic heart assist pump in the actual dimensions. This method can be used to transform results in pixels to dimensions in the real world in millimetres. This was a continuation of works on the use of image processing and analysis techniques for calculating the stroke volume of an artificial ventricle.

In the study the appropriate equations to transformation of the $\mathrm{x}-, \mathrm{y}$ - and z-coordinates were analyzed and determined. These equations allow the user to obtain a measurement grid which represents the shape of the membrane dimensioned in the actual 3-dimensional space.

The usefulness of the determined transformation equations was confirmed by the conducted measurements for two extreme states of the membrane.

The developed method will enable to determine the stroke volume of the artificial ventricle using the numerical integration method.

\section{REFERENCES}

[1] K. Murawski, "Method of measuring the distance using one camera", Patent Application: P.408076, 2014. (in Polish).

[2] K. Murawski, M. Murawska, T. Pustelny, "The system and method of determining the shape of the membrane pneumatic pump of extracorporeal heart assist device", Patent Application: nr P.414104, 2015. (in Polish).

[3] K. Murawski, "Measurement of membrane displacement using a motionless camera", Acta Phys. Pol. A, 128, 1, 2015, 10 - 14. DOI: 10.12693/APhysPolA.128.10.

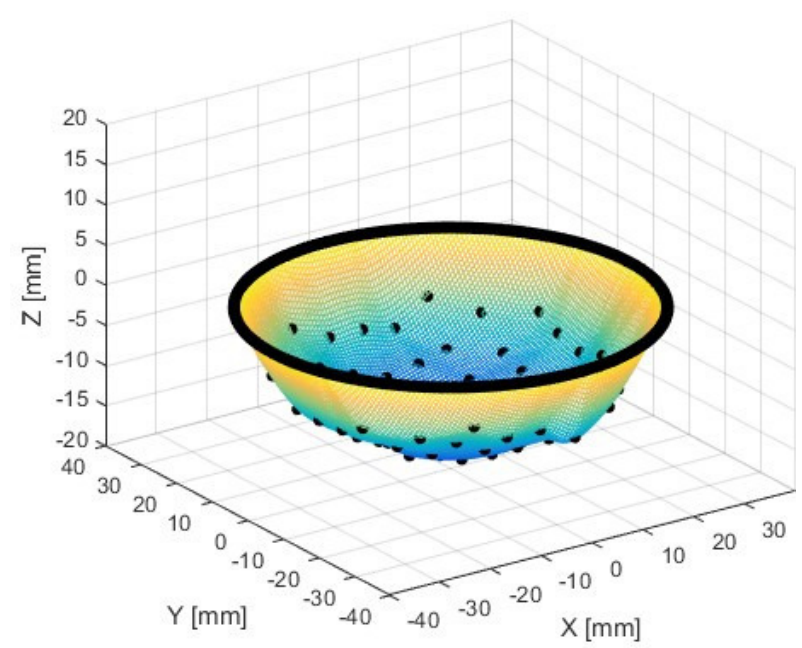

Fig. 16 The mapped shape of concave membrane from the measurements

[4] K. Murawski, "Measurement of membrane displacement with a motionless camera equipped with a fixed focus lens", Metrology and Measurement Systems, 22, 1, 2015, 69 - 78. DOI: 10.1515/mms2015-0011.

[5] K. Murawski, A. Arciuch, T. Pustelny, "Studying the influence of object size on the range of distance measurement in the new Depth From Defocus method", 2016 Federated Conference on Computer Science and Information Systems (FedCSIS), Gdansk, 2016, pp. 817822. DOI: 10.15439/2016F136.

[6] J. Sarna, R. Kustosz, E. Woźniewska, M. Gonsior, A. Jarosz, K. Szymańska, D. Hansel, E. Krzak, Program „Polskie Sztuczne Serce” Sojusz Medycyny, Nauki i Techniki, ISBN 978-83-63310-16-5, 2013, (in Polish).

[7] P. Gibinski, G. Konieczny, E. Maciak, Z. Opilski, T. Pustelny, "Acoustic device for measuring instantaneous blood volume in cardiac support chamber i.e. pneumatic heart assist driving chamber, has sensor supporting heart in openings, and audio amplifier connected with volume unit of blood-cell support", Patent Number(s): PL394074-A1, 2011.

[8] G. Konieczny, T. Pustelny, P. Marczyński, “Optical sensor for measurements of the blood chamber volume in the POLVAD Prosthesis - static measurements", Acta Phys. Pol. A, 124, 3, 2013, 479 - 482. DOI: 10.12693/APhysPolA.124.479.

[9] L. Grad, K. Murawski, T. Pustelny, "Measuring the stroke volume of the pneumatic heart prosthesis using an artificial neural network", Proc. SPIE 10034, 11th Conference on Integrated Optics: Sensors, Sensing Structures, and Methods, 2016; DOI: 10.1117/12.2243952.

[10] K. Murawski, T. Pustelny, L. Grad, M. Murawska, "Estimation of the blood volume in pneumatically controlled ventricular assist device by vision sensor and image processing technique", Proc. 21st International Conference on Methods and Models in Automation and Robotics (MMAR), 2016; DOI: 10.1109/MMAR.2016.7575115.

[11] W. Sulej, L. Grad, K. Murawski, "The technique of accuracy measurement of membrane shape mapping of an artificial ventricle", Proc. SPIE 10455, 12th Conference on Integrated Optics: Sensors, Sensing Structures, and Methods, 2017; DOI: 10.1117/12.2280806.

[12] L. Grad, K. Murawski, W. Sulej, "Research to improve the accuracy of determining the stroke volume of an artificial ventricle using the wavelet transform", Proc. SPIE 10455, 12th Conference on Integrated Optics: Sensors, Sensing Structures, and Methods, 2017; DOI: $10.1117 / 12.2280804$. 